\title{
Gender-specific cephalometric features related to obesity in sleep apnea patients: trilogy of soft palate-mandible-hyoid bone
}

Seok Hyun Cho' ${ }^{1}$ Jae-Yun Jeon ${ }^{2}$, Kun-Soo Jang ${ }^{2}$, Sang Yoon Kim³ ${ }^{3,4}$, Kyung Rae Kim¹, Seungho Ryu ${ }^{5}$ and Kyung-Gyun Hwang ${ }^{2^{*}}$ (D)

\begin{abstract}
Background: The aim of this study is to investigate the relationship between gender-specific and obesity-related airway anatomy in patients with obstructive sleep apnea (OSA) by using cephalometric analyses.

Methods: We retrospectively evaluated 206 patients with suspected OSA undergoing polysomnography and anthropometric measurements such as body mass index, neck circumference, and waist-hip ratio. We checked lateral cephalometry to measure tissue landmarks including angle from A point to nasion to B point (ANB), soft palate length (SPL), soft palate thickness (SPT), retropalatal space (RPS), retrolingual space (RLS), and mandibular plane to hyoid (MPH).

Results: Male with OSA showed significantly increased SPL $(P=.006)$ compared with controls. SPL and MPH had significant correlation with apnea-hypopnea index (AHI) and central obesity. Female with OSA showed significantly increased ANB $(P=.013)$ and SPT $(P=.004)$ compared with controls. The receiver operating characteristic curves revealed that SPT in male and ANB and SPT in female were significant in model 1 (AHI $\geq 5$ ) and model 2 (AHI $\geq 15$ ). MPH was also significant for male in model 2.

Conclusion: Male and female with OSA had distinct anatomic features of the upper airway and different interactions among soft palate, mandible, and hyoid bone.
\end{abstract}

Keywords: Central obesity, Cephalometry, Gender, Sleep disorders, Airway

\section{Background}

Obstructive sleep apnea (OSA) refers to sleep-disordered breathing showing repetitive episodes of upper airway collapse during sleep [1]. The risk factors for OSA have been reported as old age, male, smoker, alcohol use, obesity, and craniofacial abnormality [2-6]. Therefore, the pathogenesis of OSA may be related to altered anatomy (small box) and physiology (increased collapsibility) of the upper airway. However, the exact mechanism of OSA has not been fully elucidated. Our previous study demonstrated that a predictor variable neck circumference $(\mathrm{NC})$ is reliable anthropometry for male with OSA [7]. However, it is

\footnotetext{
* Correspondence: hkg@hanyang.ac.kr

${ }^{2}$ Department of Dentistry/Oral and Maxillofacial Surgery, School of Medicine, Hanyang University, 222-1 Wangshimniro, Seongdong-gu, Seoul 133-792, Korea

Full list of author information is available at the end of the article
}

not clear how this localized obesity in the neck alters the anatomy of the upper airway.

Polysomnography (PSG) is the gold standard test to diagnose the presence of OSA and to estimate its severity. And, apnea-hypopnea index (AHI) criteria with the mean and lowest $\mathrm{SpO}_{2}$ are used for treatment planning. However, they do not provide the anatomic locations of obstruction.

Most sleep surgeons perform upper airway imaging studies such as awake and sleep endoscopy, lateral cephalometry, fluoroscopy, computed tomography (CT), or magnetic resonance imaging (MRI) in order to determine surgical versus medical (CPAP, weight control, and oral appliances) management for OSA. The lateral cephalometry is a traditional dental radiograph that shows twodimensional structures of bony and soft tissues. Riley et al. [8] reported anatomical characteristics of OSA patients 
with cephalometric analysis in 1983, and thereafter, a series of analyses on OSA patients have been performed to validate its sensitivity as a diagnostic tool for OSA [912]. However, the diagnostic values of lateral cephalometry in previous studies remained uncertain. Our hypothesis was that compared with females, males with OSA may have different characteristics of the upper airway which can be influenced by genetics (sex) and environments (central obesity).

This study measured the anthropometry (body mass index (BMI), neck circumference (NC), waist circumference (WC), and waist-hip ratio (WHR)) and obtained a full-night PSG and lateral cephalometry. The aim of this study was to evaluate the relationships between the upper airway anatomy (lateral cephalometry) and anthropometry in patients with OSA. In addition, we investigated the gender-specific and obesity-related anatomy of the upper airway in OSA patients to aid the treatment approach and management.

\section{Methods \\ Patients}

There were 288 patients with OSA symptoms (e.g., excessive daytime sleepiness, loud snoring, or observed apnea episodes) who visited the sleep clinic at University Hospital. Exclusion criteria were as follows: (1) patient younger than 18 years $(n=5)$; (2) non-Asian patients $(n=10)$; (3) patients who refused to undergo PSG $(n=22)$; and lateral cephalometry $(n=45)$. A total of 206 patients were included in this study. The symptom questionnaire was used to assess daytime sleepiness (Epworth Sleepiness Scales (ESS)) and sleep quality (Pittsburgh Sleep Quality Index (PSQI)). Detailed medical history was obtained, and anthropometry (BMI, NC, WC, and WHR) was measured prior to PSG. All study protocol was approved by the local Institutional Review Board, and all subjects provided written informed consent (HY-2019-01-007-001).

\section{Polysomnography}

In-laboratory PSG was performed per standard clinical guideline s[13] and included full EEG, EOG, chin EMG, leg EMG, ECG, airflow recorded with nasal/oral thermistor and nasal/oral cannula, pulse oximetry, and body position. According to the American Academy of Sleep Medicine (AASM) Sleep Apnea Definitions Task Force defined in 2012, apnea was scored when there is a drop in the peak signal excursion by $\geq 90 \%$ of pre-event baseline using an oronasal thermal sensor lasting $\geq 10 \mathrm{~s}$. Hypopneas were scored when (1) the peak signal excursions drop by $\geq 30 \%$ of preevent baseline using nasal pressure lasting $\geq 10 \mathrm{~s}$ and (2) there is $\geq 3 \%$ oxygen desaturation from pre-event baseline or the event is associated with an arousal. The AHI was calculated as the number of apnea plus hypopnea per hour of sleep [14]. The cutoff point for OSA was an AHI $\geq 5$.

\section{Cephalometric measurements}

Lateral cephalometry was taken after performing PSG and was taken using a standard protocol. All patients were positioned such that the FH plane was parallel to ground level with upper and lower lips, tongue relaxed, and teeth in centric occlusion while holding the breath at the end of the expiratory phase. The length and angular parameters of bony and soft tissue landmarks were measured using the picture archiving and communication system (PACS, Piview STAR, INFINITT, Korea) and analyzed the data using the V-ceph 5.0 software (Cybermed Inc., Seoul, Korea). Cephalometric analyses were corrected for cranio-cervical inclination in order to standardize the data and correction of head position [15]. SNA (angle from sella (S) to nasion (N) to A point), SNB (angle from $\mathrm{S}$ to $\mathrm{N}$ to $\mathrm{B}$ point), and ANB (angle from $\mathrm{A}$ point to $\mathrm{N}$ to $\mathrm{B}$ point) were measured to obtain protrusion angles of maxilla and mandible from the cranium. Posterior airway space (PAS) was measured at two locations: retropalatal space (RPS) and retrolingual space (RLS). Anatomy of the soft palate was measured with its length (SPL) and thickness (SPT). The linear distance along the perpendicular plane from the hyoid bone mandibular plane (MPH) was measured (Table 1 and Fig. 1).

\section{Statistical analysis}

All measured values were presented as mean (standard deviation) for continuous variables and as frequencies and percentages for categorical variables. Student's $t$ test was used to assess the differences among continuous variables, and the categorical variables between genders were analyzed by chi-square test. Pearson's correlation coefficient was used to determine the correlations between AHI and anthropometric and cephalometric data. Cutoff values and its sensitivity, specificity, and positive and negative predictive values were calculated for each gender. All cephalometric parameters were compared in terms of

\section{Table 1 Cephalometric measurements}

\begin{tabular}{|c|c|}
\hline Variable & Definition \\
\hline SNA & Angle from sella to nasion to A point \\
\hline SNB & Angle from sella to nasion to B point \\
\hline ANB & Angle from $A$ point to nasion to $B$ point \\
\hline $\begin{array}{l}\text { SPL } \\
\text { (soft palate length) }\end{array}$ & Distance from PNS to tip of the soft palate \\
\hline $\begin{array}{l}\text { SPW } \\
\text { (soft palate width) }\end{array}$ & $\begin{array}{l}\text { Widest width along perpendicular line to PNS to } \\
\text { tip of soft palate }\end{array}$ \\
\hline $\begin{array}{l}\text { RPS } \\
\text { (retropalatal space) }\end{array}$ & $\begin{array}{l}\text { Shortest distance from soft palate to posterior } \\
\text { pharyngeal wall }\end{array}$ \\
\hline $\begin{array}{l}\text { RLS } \\
\text { (retrolingual space) }\end{array}$ & $\begin{array}{l}\text { Shortest distance from tongue base to posterior } \\
\text { pharyngeal wall }\end{array}$ \\
\hline $\mathrm{MPH}$ & $\begin{array}{l}\text { Linear distance along the perpendicular plane from } \\
\text { Hyoid to mandibular plane }\end{array}$ \\
\hline
\end{tabular}




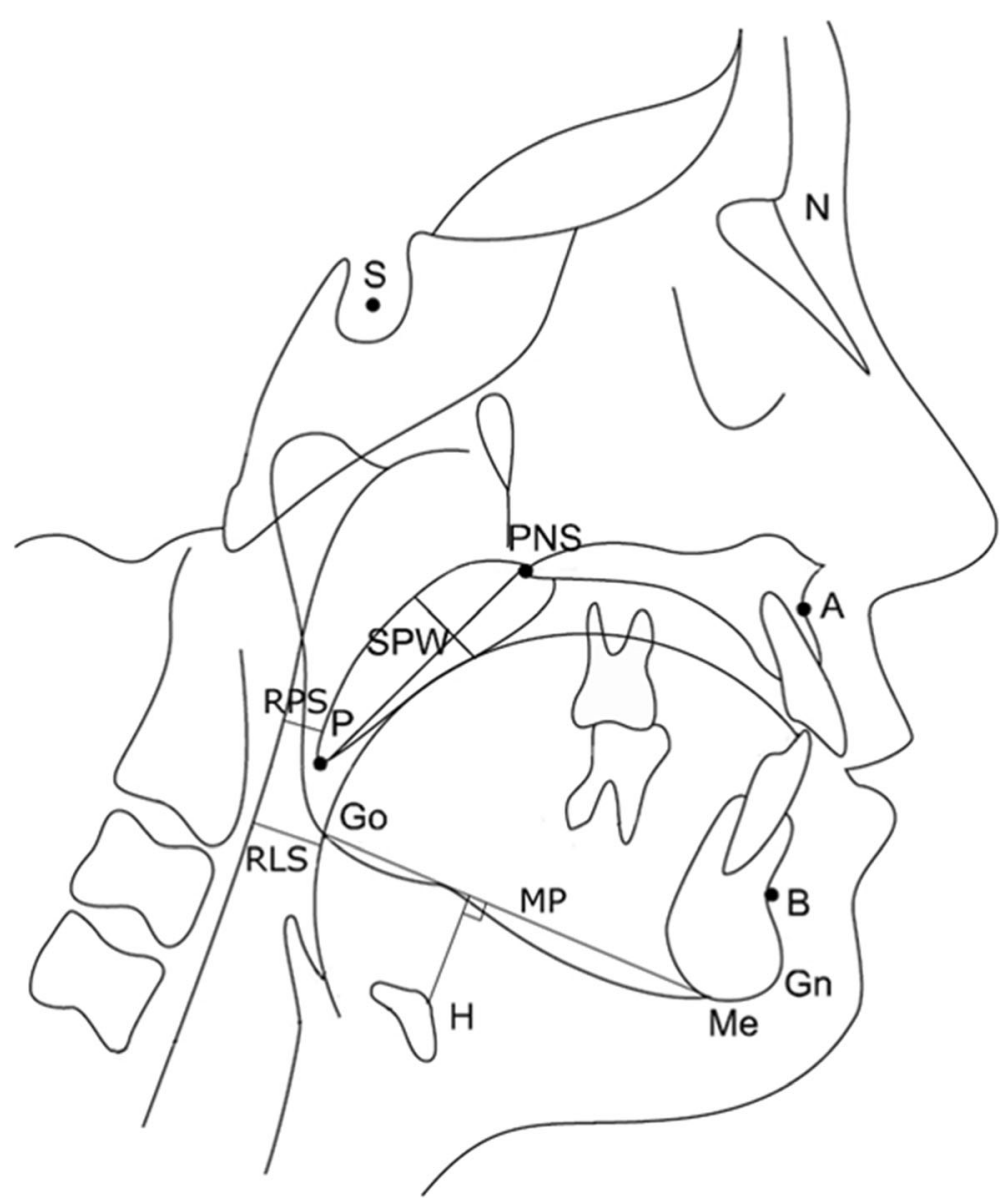

Fig. 1 Linear and angular measurements for bony and soft tissue framework in lateral cephalometry. S (sella), midpoint of the fossa hypophysealis; N (nasion), anterior point at the frontonasal suture; $\mathrm{A}$, the deepest anterior point in the concavity of the anterior maxilla; $\mathrm{B}$, the deepest anterior point in the concavity of the anterior mandible; PNS (posterior nasal spine), the most posterior point on the nasal spine; Go (gonion), a mid-point at the gonial angle located by bisecting the posterior and inferior borders of the mandible; Gn (gnathion), the most anteroinferior point on the chin bone; Me (menton), the most inferior point on the chin bone; $\mathrm{P}$, the inferior tip of the soft palate; $\mathrm{H}$, the most anterosuperior point on the hyoid bone; MP (mandibular plane), a tangent line from Me to the inferior mandibular border

their receiver operating characteristic (ROC) curves. This method compares the diagnostic properties of the test by expressing sensitivity as a function of $1-$ specificity. Results were considered significant at a value of $P<.05$ and with a confidence interval (CI) of $95 \%$. All analyses were performed with Statistical Package for the Social Sciences (SPSS) version 18.0 (SPSS Inc., Chicago, IL, USA).

\section{Results}

Two hundred six subjects were enrolled in the study and Table 2 shows their clinical characteristics with anthropometry, cephalometry, and polysomnography results. OSA groups were older than corresponding control groups without any differences in ESS and PSQI. In male with OSA, BMI, NC, and WHR were significantly elevated than controls and showed significant correlations with AHI (Table 3). However, WHR was the only parameter with higher values in female with OSA and also had a significant correlation with AHI. All patients with OSA showed significantly worsen $\mathrm{AHI}$ and lowest nadir $\mathrm{SpO}_{2}$ than controls.

\section{Gender-specific characteristics of the upper airway anatomy in OSA}

Male and female with OSA had different cephalometric profiles (Table 2). In male, SPL $(P=.006)$ was significantly increased in the OSA group but there was no difference in other parameters. However, in female, ANB $(P=.013)$ and SPT $(P=.004)$ were significantly higher in the OSA group but there was no difference in other parameters.

\section{Gender differences in associations between the upper airway anatomy and $\mathrm{AHI}$}

Male and female showed different associations between the upper airway anatomy (lateral cephalometry) and AHI (Table 3). In male, SPL $(r=.267, P=.001)$ and $\mathrm{MPH}(r=.292, P=.001)$ showed significant correlations 
Table 2 Gender effects on cephalometric differences between OSA and control patients

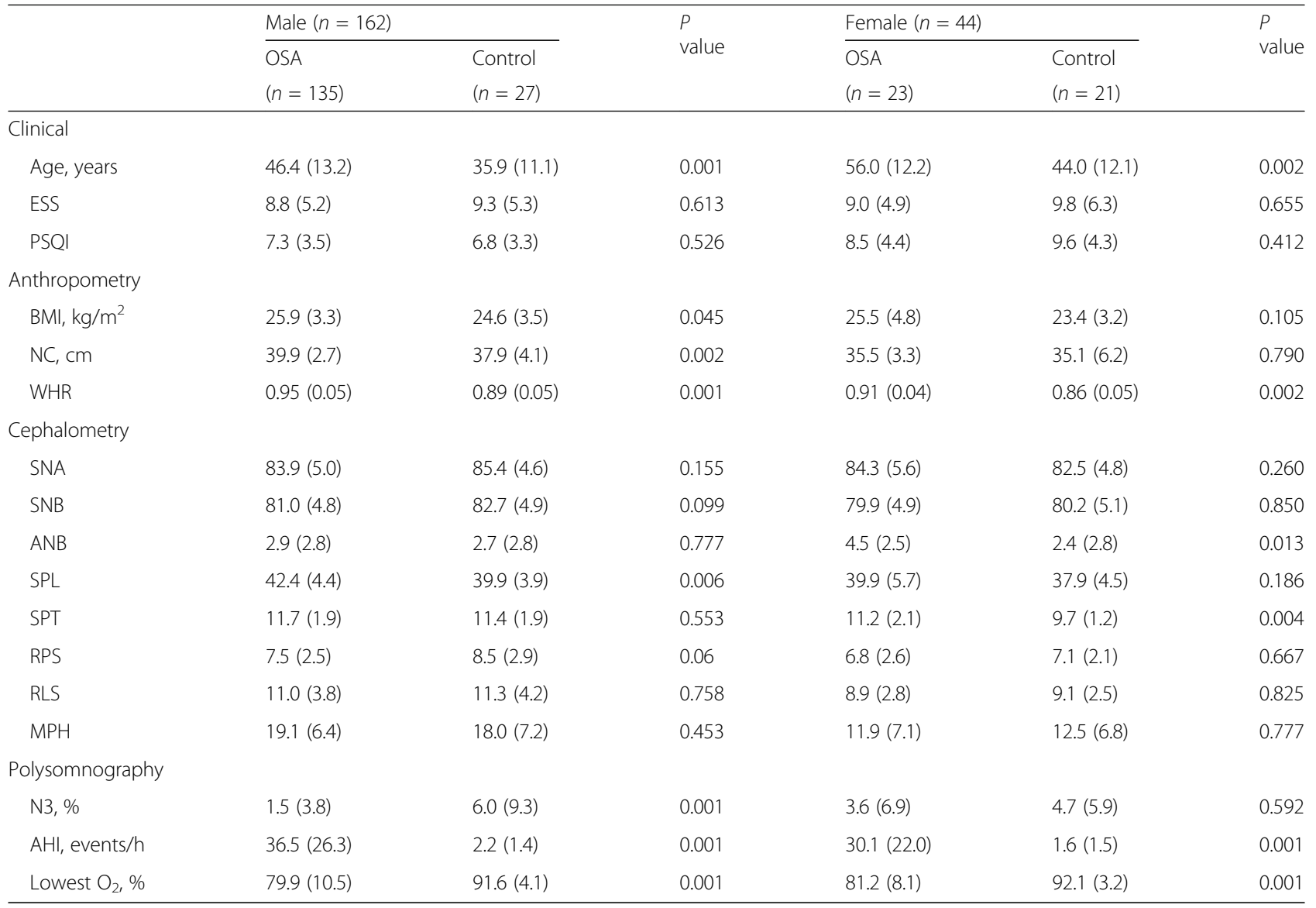

All values are reported as mean (SD). OSA, obstructive sleep apnea; ESS Epworth Sleepiness Scale, PSQI Pittsburgh Sleep Quality Index, NC neck circumference, WHR waist-hip ratio, SE sleep efficiency, N3 deep sleep, AHI apnea-hypopnea index

Table 3 Correlation of anthropometric and cephalometric parameters with $\mathrm{AHI}$

\begin{tabular}{llll}
\hline Parameters & All subjects & Male & Female \\
\hline Anthropometry & & & \\
BMI & $0.356^{* *}$ & $0.368^{* *}$ & 0.240 \\
NC & $0.361^{* *}$ & $0.387^{* *}$ & 0.103 \\
WHR & $0.428^{* *}$ & $0.384^{* *}$ & $0.397^{* *}$ \\
Cephalometry & & & \\
ANB & 0.079 & 0.042 & $0.384^{*}$ \\
SPL & $0.284^{* *}$ & $0.267^{* *}$ & 0.141 \\
SPT & $0.234^{* *}$ & 0.150 & $0.386^{* *}$ \\
RPS & -0.048 & -0.053 & -0.208 \\
RLS & $0.137^{*}$ & 0.108 & -0.024 \\
MPH & $0.339^{* *}$ & $0.292^{* *}$ & 0.253 \\
\hline
\end{tabular}

${ }^{*} P<0.05 ;{ }^{*} P<0.01$. Numbers are Pearson's correlation coefficient ( $r$ values). $A H I$ apnea-hypopnea index, $B M I$ body mass index, $N C$ neck circumference, WHR waist-hip ratio, $A N B$ angle from A point to nasion to B point, SPL soft palate length, SPT soft palate thickness, RPS retropalatal space, $R L S$ retrolingual space, $M P H$ mandibular plane to hyoid with AHI. However, in female, SPT $(r=.386, P=.01)$ showed a significant correlation with AHI.

\section{Gender-specific effects of central obesity on the upper airway anatomy}

Central obesity had different impacts on the upper airway anatomy measured with lateral cephalometry (Table 4). In male, NC showed significant correlations with SPL $(r=.208$, $P=.008)$, SPT $(r=.3, P=.001)$, RLS $(r=.168, P=.033)$, and $\mathrm{MPH}(r=.161, P=.042)$. And WHR showed significant correlation with SPT $(r=.211, P=.007)$ and $\mathrm{MPH}(r=.162, P$ $=.04)$. However, in female, none of the cephalometric indexes had any correlations with NC or WHR.

\section{Cutoff values and areas under the ROC curve of lateral cephalometry in male and female with OSA}

In male and female, ROC curves were constructed for each cephalometric factor to evaluate their diagnostic power to OSA and calculated cutoff values (Fig. 2). Table 5 gives the areas under the ROC curve for cephalometric variables and their significance in predicting OSA. In model 1 ( $\mathrm{AHI} \geq 5$ ), the areas under the ROC 
Table 4 Effect of central obesity (anthropometry) on the upper airway anatomy (cephalometry)

\begin{tabular}{llll}
\hline Parameters & All subjects & Male & Female \\
\hline Correlation with NC & & & \\
ANB & -0.120 & -0.124 & 0.014 \\
SPL & $0.197^{* *}$ & $0.208^{* *}$ & -0.151 \\
SPT & $0.305^{* *}$ & $0.300^{* *}$ & 0.075 \\
RPS & 0.022 & -0.028 & -0.037 \\
RLS & $0.168^{*}$ & $0.168^{*}$ & -0.194 \\
MPH & $0.314^{* *}$ & $0.161^{*}$ & 0.231 \\
Correlation with WHR & & \\
ANB & -0.046 & -0.066 & 0.210 \\
SPL & $0.177^{*}$ & 0.126 & -0.033 \\
SPT & $0.258^{* *}$ & $0.211^{* *}$ & 0.104 \\
RPS & 0.067 & 0.047 & -0.048 \\
RLS & $0.186^{* *}$ & 0.136 & 0.025 \\
MPH & $0.245^{* *}$ & $0.162^{*}$ & 0.002 \\
\hline
\end{tabular}

${ }^{*} P<0.05 ;{ }^{* *} P<0.01$. Numbers are Pearson's correlation coefficient ( $r$ values). $N C$ neck circumference, WHR waist-hip ratio, $A N B$ angle from A point to nasion to $B$ point, SPL soft palate length, SPT soft palate thickness, RPS retropalatal space, $R L S$ retrolingual space, $M P H$ mandibular plane to hyoid

curve for male with OSA were significant for SPL $(P=$ .005). In female, the areas under the ROC curve for OSA were significant for SPT $(P=.001)$. In model 2 (AHI $\geq$ $15)$, both SPL $(P=.004)$ and MPH $(P=.001)$ were significant for male with OSA. However, in female, SPT $(P$ $=.003)$ remained significant for OSA.

\section{Discussion}

This study investigated the anatomic variants of the upper airway (lateral cephalometry) related to sex and central obesity in Asian patients with OSA. The different phenotypes of OSA determined by sex and central obesity may permit us to make appropriate therapeutic plans for OSA patients. The key findings of this study were as follows: (1) Soft palate was found to be the main target of OSA and its anatomic changes show significant correlations with AHI. (2) Phenotypes of soft palate were different between sexes: increased length for male and increased thickness for female. (3) Only male OSA patients showed clear associations between central obesity and the upper airway anatomy. (4) MPH was a malespecific parameter especially for moderate and severe OSA and showed a significant correlation with both AHI and central obesity.

Male with OSA showed typical characteristics of central obesity with increased NC and WHR. There have been many studies demonstrating the altered neck anatomy in male with OSA including increased neck circumference (anthropometry) and parapharyngeal fat deposition (CT or MRI) [16-18]. Therefore, it is reasonable to state that central obesity (thick neck) in male with OSA may cause the altered anatomy of the upper airway. To illustrate OSA-related upper airway anatomy, a total of eight well-known parameters on lateral cephalometry were used for craniofacial anatomy (SNA, SNB, and ANB), upper airway (SPL, SPT, and RPS), and lower airway (RLS and MPH). Lateral cephalometry does not show that the volume of airway and cephalometric analysis was affected by the head position. Nevertheless, it has been used in maxillofacial deformity analysis and orthodontic diagnosis. The lateral cephalometry has been used for its simplicity and economical method for measuring anatomical structure related to the airway. In this study, we standardized the data by correcting the cranio-cervical inclination in order to obtain accurate measurements of parameters of the airway. This study clearly demonstrated that the upper airway anatomy measured with lateral cephalometry had different characteristics and associations with AHI and central obesity, depending on sex.

SNA, SNB, and ANB were used to evaluate the anterior-posterior position of maxilla and mandible to cranial base on lateral cephalometry. Retrognathia is well-known anatomic phenotype affecting the pathogenesis of OSA [19-22]. Lowe et al. [21] showed that patients with OSA had the smaller and posteriorly positioned mandible which decreased the overall airway space on lateral cephalometry. In contrast, others reported that there was no difference in upper airway volume based on the intermaxillary relationship using cone beam computed tomography (CBCT) [23]. However, our study demonstrated that mandible was positioned posteriorly in female with OSA (increased ANB) which was independent with obesity and had significant correlations with AHI. Thus, retrognathia is an important anatomic phenotype affecting the presence and severity of OSA in female.

There are many reports using lateral cephalometry to investigate the influence of soft tissues on OSA. Previous studies reported that increased soft palate length and thickness are associated with OSA on lateral cephalometry [21, 24-27]. In the study targeting 62 males, Yu et al. [28] also reported that longer SPL was associated with higher prevalence of OSA. In contrast, Cillo et al. [29] reported that there were no significant changes in SPL in OSA. However, in this study, we found that male with OSA had a different phenotype of the soft palate compared with female with OSA. Male with OSA had significantly increased SPL which showed significant association with AHI and NC. Alternatively, female with OSA had significantly increased SPT which showed significant association with AHI but not with central obesity (NC and WHR). Therefore, the soft palate may become elongated in conjunction with the weight gain 

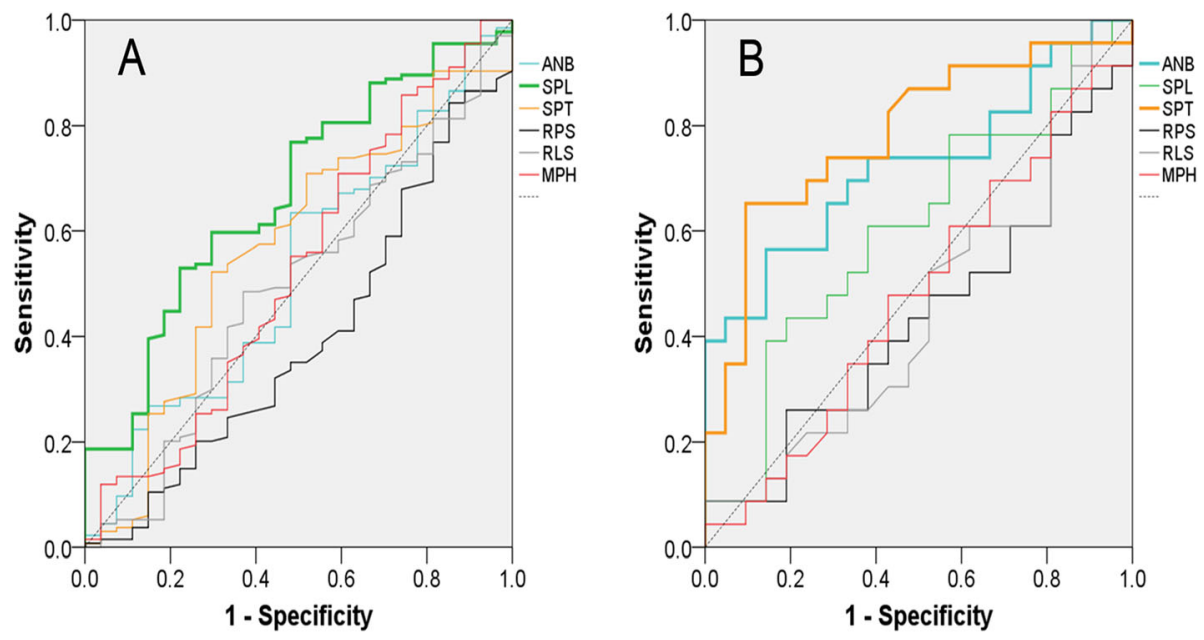

Fig. 2 Receiver operating characteristic curves in male (a) and female (b) for angle from sella to nasion to A point (ANB), soft palate length (SPL), soft palate thickness (SPT), retropalatal space (RPS), retrolingual space (RLS), linear distance along the perpendicular plane from hyoid to mandibular plane (MPH)

which can aggravate the upper airway narrowing in male. However, female may have an intrinsic variance of SPT which is independent with central obesity. Female with thick palate may have increased risk for the development of OSA (Fig. 3).

Retro-palatal and retro-lingual spaces are the most common sites of upper airway narrowing in OSA [30,

Table 5 Comparison of areas under the receiver operating characteristic curves in male and female

\begin{tabular}{|c|c|c|c|c|}
\hline & \multicolumn{2}{|l|}{ Male } & \multicolumn{2}{|c|}{ Female } \\
\hline & AUC & $P$ value & $A \cup C$ & $P$ value \\
\hline \multicolumn{5}{|c|}{ Model 1 (AHI $\geq 5)$} \\
\hline ANB & 0.519 & 0.751 & 0.725 & $0.011^{*}$ \\
\hline SPL & 0.670 & $0.005^{* *}$ & 0.605 & 0.235 \\
\hline SPT & 0.568 & 0.265 & 0.784 & $0.001^{* *}$ \\
\hline RPS & 0.396 & 0.087 & 0.437 & 0.474 \\
\hline RLS & 0.493 & 0.912 & 0.452 & 0.589 \\
\hline $\mathrm{MPH}$ & 0.527 & 0.656 & 0.477 & 0.796 \\
\hline \multicolumn{5}{|c|}{ Model 2 (AHI $\geq 15)$} \\
\hline ANB & 0.547 & 0.310 & 0.736 & $0.009^{* *}$ \\
\hline SPL & 0.635 & $0.004^{* *}$ & 0.498 & 0.981 \\
\hline SPT & 0.568 & 0.144 & 0.772 & $0.003^{* *}$ \\
\hline RPS & 0.481 & 0.675 & 0.461 & 0.090 \\
\hline RLS & 0.549 & 0.294 & 0.511 & 0.089 \\
\hline $\mathrm{MPH}$ & 0.648 & $0.001^{* *}$ & 0.523 & 0.094 \\
\hline
\end{tabular}

${ }^{*} P<0.05 ;{ }^{*} P<0.01$. AUC area under the ROC curve, $A H I$ apnea-hypopnea index, $A N B$ angle from $A$ point to nasion to B point, SPL soft palate length, SPT soft palate thickness, RPS retropalatal space, $R L S$ retrolingual space, $M P H$ mandibular plane to hyoid
31]. Dynamic studies such as drug-induced sleep endoscopy (DISE) also reported that there are isolated or multiple sites of upper airway collapse in OSA [32]. However, in our cephalometric study, there was no difference in RPS and RLS between OSA and controls regardless of the sex. Moreover, they did not show any correlation with AHI and central obesity. Thus, we concluded that 2-dimensional analysis of the upper airway space may have limitations to represent OSA. To investigate the space anatomy, upper airway endoscopy may be advantageous to show 3-dimensional views and dynamics in OSA.

The position of the hyoid bone is related to the retro-lingual space, and lower hyoid indicates the narrowing of RLS and also is associated with weak tonicity of the genioglossus muscle. De berry et al. [33] reported that the hyoid bone in the lower position could displace the base of the tongue further downward leading to the airway obstruction of hypopharynx more easily. However, in our study, there was no difference in $\mathrm{MPH}$ between OSA and controls regardless of sex. By subgroup analysis, $\mathrm{MPH}$ in male showed significant associations with $\mathrm{AHI}$ and central obesity (NC and WHR). Moreover, ROC curves showed that $\mathrm{MPH}$ is an anatomic factor representing the moderate-to-severe OSA in male. Whittle et al. [16] reported that male had larger soft tissue volume around the airway compared with female which is consistent with our study. Thus, both intrinsic soft tissue volumes of the upper airway and obesityrelated central fat deposition (NC) may affect the $\mathrm{MPH}$ in male. By comparison, $\mathrm{MPH}$ in female did not show any relationship with $\mathrm{AHI}$ and obesity, and 


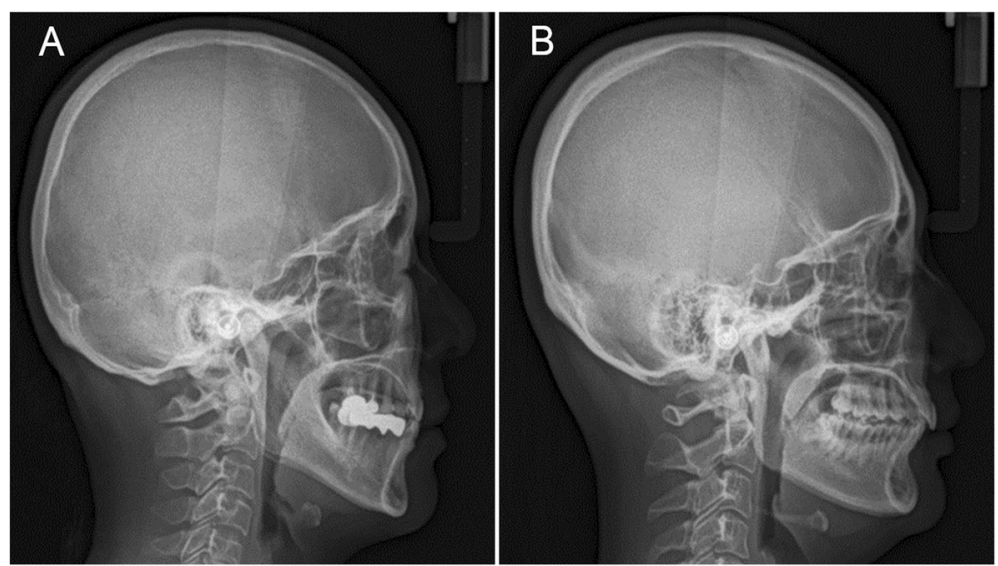

Fig. 3 Representative views of lateral cephalometry show the different morphology of the soft palate, mandible, and hyoid bone between male (a) and female (b) with obstructive sleep apnea

therefore, the narrowing of RLS may be not involved in the pathogenesis of female OSA.

The authors acknowledge the weaknesses and limitations of our study. The lateral cephalometry was taken in a standing position and awake state which does not represent accurate measurements of the upper airway in the sleeping state. Also, the number of female was relatively fewer than male. Despite the above-mentioned limitations, our study clearly demonstrated the role of gender on the upper airway anatomic variation and its association with central obesity.

\section{Conclusion}

Phenotypes of the upper airway in OSA differ with the sex. Increased SPL and MPH contributed to OSA in male. However, retrognathia and increased SPT are involved in the pathogenesis of OSA in female. Altered upper airway anatomy is significantly associated with central obesity in male, but these interactions were not found in female.

Male and female may have distinct upper airway anatomy, and different risk factors are involved in the pathogenesis of OSA. Central obesity may increase SPL and $\mathrm{MPH}$ and, therefore, aggravate the upper and lower airway collapse in OSA. By comparison, developmental retrognathia and thick soft palate independent with obesity may affect OSA in female.

Our study demonstrated the hidden and useful features of lateral cephalometry for the evaluation of the upper airway in OSA. When considering sex-specific therapeutic plan for OSA, obesity-susceptible upper airway narrowing may indicate the effectiveness of weight control for male with OSA. And retrognathia and thick palate in female may predict the lack of success rate of the palate surgery without tonsillar hypertrophy. Further study will be necessary to demonstrate the values of these anatomy-based treatment plans for OSA.

\section{Abbreviations}

ANB: A point to nasion to B point; BMI: Body mass index; CPAP: Continuous positive airway pressure; MPH: Mandibular plane to hyoid; OSA: Obstructive sleep apnea; PSG: Polysomnography; RLS: Retrolingual space;

RPS: Retropalatal space; SPL: Soft palate length; SPT: Soft palate thickness

\section{Acknowledgements}

This work was supported by the National Research Foundation of Korea Grant funded by the Korean Government (NRF-2017 M3A9F 1027928).

\section{Authors' contributions}

SH, JY, KS, and SY carried out the experiments and wrote the manuscript. KG and KR conceived this experiment and reviewed this manuscript. SR analyzed the data. All authors were involved in writing the paper. All authors read and approved the final manuscript.

\section{Funding}

This study received no specific grant from any funding agency in the public, commercial, or not-for-profit sectors.

\section{Availability of data and materials}

Data sharing is not applicable to this article as no data sets were generated or analyzed during the current study.

\section{Ethics approval and consent to participate}

This case report was reviewed by the Institutional Review Board (IRB) of Hanyang University Hospital and was approved from deliberation (HY-201901-007-001).

\section{Consent for publication}

This manuscript does not contain any individual person's identifier

\section{Competing interests}

The authors declare that they have no competing interests.

\section{Author details}

'Department of Otorhinolaryngology-Head and Neck Surgery, School of Medicine, Hanyang University, Seoul, Korea. ${ }^{2}$ Department of Dentistry/Oral and Maxillofacial Surgery, School of Medicine, Hanyang University, 222-1 Wangshimniro, Seongdong-gu, Seoul 133-792, Korea. ${ }^{3}$ Department of Oral and Maxillofacial Surgery, Massachusetts General Hospital, Harvard Medical School, Boston, USA. ${ }^{4}$ Vienna, USA. ${ }^{5}$ Department of Occupational Medicine, Kangbuk Samsung Hospital, School of Medicine, Sungkyunkwan University, Seoul, Korea. 
Received: 17 July 2019 Accepted: 27 November 2019

Published online: 11 December 2019

\section{References}

1. Sleep-related breathing disorders in adults: recommendations for syndrome definition and measurement techniques in clinical research (1999) The report of an American Academy of Sleep Medicine Task Force. Sleep. 22: 667-689

2. Bixler EO, Vgontzas AN, Ten Have T, Tyson K, Kales A (1998) Effects of age on sleep apnea in men: I. Prevalence and severity. Am J Respir Crit Care Med 157:144-148

3. Dancey DR, Hanly PJ, Soong C, Lee B, Shepard J Jr, Hoffstein V (2003) Gender differences in sleep apnea: the role of neck circumference. Chest 123:1544-1550

4. Romero-Corral A, Caples SM, Lopez-Jimenez F, Somers VK (2010) Interactions between obesity and obstructive sleep apnea: implications for treatment. Chest 137:711-719

5. Jamieson A, Guilleminault C, Partinen M, Quera-Salva MA (1986) Obstructive sleep apneic patients have craniomandibular abnormalities. Sleep. 9:469-477

6. Jang SI, Ahn J, Paeng JY, Hong J (2018) Three-dimensional analysis of changes in airway space after bimaxillary orthognathic surgery with maxillomandibular setback and their association with obstructive sleep apnea. Maxillofac Plastic Reconstruc Surg 40:33-44

7. Lim YH, Choi J, Kim KR, Shin J, Hwang KG, Ryu S, Cho SH (2014) Sex-specific characteristics of anthropometry in patients with obstructive sleep apnea: neck circumference and waist-hip ratio. Ann Otol Rhinol Laryngol 123:517-523

8. Riley R, Guilleminault C, Herran J, Powell N (1983) Cephalometric analyses and flow-volume loops in obstructive sleep apnea patients. Sleep. 6:303-311

9. Guilleminault C, Riley R, Powell N (1984) Obstructive sleep apnea and abnormal cephalometric measurements. Implications for treatment. Chest. 86:793-794

10. Riley R, Guilleminault C, Powell N, Simmons FB (1985) Palatopharyngoplasty failure, cephalometric roentgenograms, and obstructive sleep apnea. Otolaryngol Head Neck Surg. 93:240-244

11. Cho HW, Kim IK, Cho HY, Seo JH, Lee DH, Park SH (2015) Retrospective study of changes in pharyngeal airway space and position of hyoid bone after mandibular setback surgery by cephalometric analysis. Maxillofac Plast Reconstruc Surg 37:38-44

12. Riley R, Powell N,(1986) Guilleminault C. Cephalometric roentgenograms and computerized tomographic scans in obstructive sleep apnea. Sleep. 9 : 514-515

13. Annals of Otology RaL.(1999) Sleep-related breathing disorders in adults: recommendations for syndrome definition and measurement techniques in clinical research. The Report of an American Academy of Sleep Medicine Task Force. Sleep. 22:667-689

14. Berry RB, Budhiraja R, Gottlieb DJ, Gozal D, Iber C, Kapur VK, Marcus CL, Mehra R, Parthasarathy S, Quan SF, Redline S, Strohl KP, Davidson Ward SL, Tangredi MM (2012) Rules for scoring respiratory events in sleep: update of the 2007 AASM Manual for the Scoring of Sleep and Associated Events. Deliberations of the Sleep Apnea Definitions Task Force of the American Academy of Sleep Medicine. J Clin Sleep Med 8:597-619

15. Anegawa E, Tsuyama H, Kusukawa J (2008) Lateral cephalometric analysis of the pharyngeal airway space affected by head posture. Int J Oral Maxillofac Surg 37:805-809

16. Whittle AT, Marshall I, Mortimore IL, Wraith PK, Sellar RJ, Douglas NJ (1999) Neck soft tissue and fat distribution: comparison between normal men and women by magnetic resonance imaging. Thorax 54:323-328

17. Isono S (2012) Obesity and obstructive sleep apnoea: mechanisms for increased collapsibility of the passive pharyngeal airway. Respirology. 17:32-42

18. Suto Y, Matsuo T, Kato T, Hori I, Inoue Y, Ogawa S, Suzuki T, Yamada M, Ohta Y (1993) Evaluation of the pharyngeal airway in patients with sleep apnea: value of ultrafast MR imaging. AJR Am J Roentgenol 160:311-314

19. Imes NK, Orr WC, Smith RO, Rogers RM.(1977) Retrognathia and sleep apnea. A life-threatening condition masquerading as narcolepsy. JAMA.237: 1596-1597

20. Tangugsorn V, Skatvedt O, Krogstad O, Lyberg T (1995) Obstructive sleep apnoea: a cephalometric study. Part I. Cervico-craniofacial skeletal morphology. Eur J Orthod 17(1):45-56

21. Lowe AA, Ozbek MM, Miyamoto K, Pae EK, Fleetham JA (1997) Cephalometric and demographic characteristics of obstructive sleep apnea: an evaluation with partial least squares analysis. Angle Orthod 67:143-153
22. Schellenberg JB, Maislin G, Schwab RJ (2000) Physical findings and the risk for obstructive sleep apnea. The importance of oropharyngeal structures. Am J Respir Crit Care Med 162:740-748

23. Di Carlo G, Polimeni A, Melsen B, Cattaneo PM (2015) The relationship between upper airways and craniofacial morphology studied in 3D. A CBCT study. Orthod Craniofac Res 18:1-11

24. Haponik EF, Smith PL, Bohlman ME, Allen RP, Goldman SM, Bleecker ER (1983) Computerized tomography in obstructive sleep apnea. Correlation of airway size with physiology during sleep and wakefulness. Am Rev Respir Dis 127:221-226

25. Tangugsorn V, Skatvedt O, Krogstad O, Lyberg T (1995) Obstructive sleep apnoea: a cephalometric study. Part II. Uvulo-glossopharyngeal morphology. Eur J Orthod 17(1):57-67

26. Lyberg T, Krogstad O, Djupesland G (1989) Cephalometric analysis in patients with obstructive sleep apnoea syndrome: II. Soft tissue morphology. J Laryngol Otol 103:293-297

27. Bacon WH, Turlot JC, Krieger J, Stierle JL (1990) Cephalometric evaluation of pharyngeal obstructive factors in patients with sleep apneas syndrome. Angle Orthod. 60:115-122

28. Yu X, Fujimoto K, Urushibata K, Matsuzawa Y, Kubo K (2003) Cephalometric analysis in obese and nonobese patients with obstructive sleep apnea syndrome. Chest 124:212-218

29. Cillo JE Jr, Thayer S, Dasheiff RM, Finn R (2012) Relations between obstructive sleep apnea syndrome and specific cephalometric measurements, body mass index, and apnea-hypopnea index. J Oral Maxillofac Surg 70:e278-e283

30. Abramson Z, Susarla S, August M, Troulis M, Kaban L (2010) Threedimensional computed tomographic analysis of airway anatomy in patients with obstructive sleep apnea. J Oral Maxillofac Surg 68:354-362

31. Yucel A, Unlu M, Haktanir A, Acar M, Fidan F (2005) Evaluation of the upper airway cross-sectional area changes in different degrees of severity of obstructive sleep apnea syndrome: cephalometric and dynamic CT study. AJNR Am J Neuroradiol. 26:2624-2629

32. Schoenberg SO, Floemer F, Kroeger H, Hoffmann A, Bock M, Knopp MV (2000) Combined assessment of obstructive sleep apnea syndrome with dynamic MRI and parallel EEG registration: initial results. Invest Radiol 35: 267-276

33. deBerry-Borowiecki B, Kukwa A, Blanks RH (1988) Cephalometric analysis for diagnosis and treatment of obstructive sleep apnea. Laryngoscope. 98:226-234

\section{Publisher's Note}

Springer Nature remains neutral with regard to jurisdictional claims in published maps and institutional affiliations.

\section{Submit your manuscript to a SpringerOpen ${ }^{\circ}$ journal and benefit from:}

- Convenient online submission

- Rigorous peer review

- Open access: articles freely available online

- High visibility within the field

- Retaining the copyright to your article

Submit your next manuscript at $>$ springeropen.com 